\title{
Exposure to volatile organic compounds and loss of pulmonary function in the elderly
}

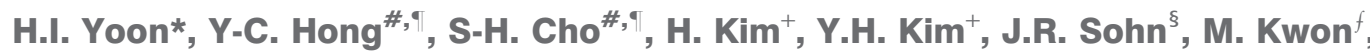 \\ S-H. Park**, M-H. Cho ${ }^{\# \#}$ and H-K. Cheong ${ }^{\oplus \uparrow}$
}

ABSTRACT: Volatile organic compounds (VOCs) are reported to cause adverse effects on pulmonary function in occupationally exposed workers. However, evidence is lacking on the effect in the general population. We hypothesised that VOCs impair pulmonary function through enhancing oxidative stress, especially in the elderly population.

A longitudinal panel study of 154 elderly people was performed in South Korea. Repeated spirometric tests were performed up to eight times on different days for each subject. We also measured urinary concentrations of metabolites of the VOC and markers of oxidative stress (malondialdehyde and 8-0xo-2'-deoxyguanosine) on the same day of spirometric tests. A mixed linear regression model was used to evaluate the association among the VOC metabolites, oxidative stress markers and spirometric tests.

We found that the urinary levels of hippuric acid and methylhippuric acid, which are metabolites of toluene and xylene, respectively, were significantly associated with reduction of forced expiratory volume in $1 \mathrm{~s} \mathrm{(FEV1),} \mathrm{FEV}_{1}$ /forced vital capacity (FVC), and forced expiratory flow at 25$75 \%$ of FVC. We also found significant associations between the metabolites of VOCs and the markers of oxidative stress. In addition, the oxidative stress markers were associated with pulmonary function parameters.

This study suggests that exposure to toluene and xylene exert a harmful effect on pulmonary function by exacerbating oxidative stress in elderly people.

KEYWORDS: Elderly, oxidative stress, pulmonary function, volatile organic compounds

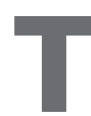
here is a growing concern over health problems related to ambient air pollution. Volatile organic compounds (VOCs) are one of the major ambient air pollutants and are emitted from diverse chemical sources in common daily use. Exposure to VOCs may cause upper and lower respiratory symptoms and contribute to the worsening of asthma. The mechanisms of VOC toxicity, however, have yet to be established, even though some experimental evidence supports oxidative stress having a role [1-3].

Decline of pulmonary function has become one of the most important issues regarding health effects of air pollution because it not only reflects the individual respiratory health status, but predicts cardiovascular morbidity and mortality in the general population [4-6].

Although the diminution of pulmonary function by exposure to ozone and particulate air pollutants has been previously reported [7-9], it is not clear whether indoor or outdoor exposure to
VOCs in ambient air contribute to the reduction of pulmonary function. Although a recent cross-sectional study suggests an association between exposure to VOCs and diminished lung function [10], evidence of a causal link is still lacking.

Therefore, we conducted a longitudinal panel study with repeated measurements to evaluate the effects of VOCs on pulmonary function and pathogenesis in the elderly, who are known to be especially susceptible to air pollution [11-13].

\section{METHODS}

\section{Study subjects}

The study population consisted of 161 elderly people aged $>60 \mathrm{yrs}$ at six day care facilities within the three different geographical regions of Seoul (three facilities), Cheongju (one facility) and Gangnung (two facilities) in South Korea, which represent a metropolitan area, an industrialised the involvement of oxidative stress in the

\section{AFFILIATIONS}

*Dept of Internal Medicine, Seoul National University Bundang Hospital,

\#Dept of Preventive Medicine, Seoul National University College of Medicine,

"Institute of Environmental Medicine, Seoul National University Medical Research Center,

${ }^{+}$Dept of Biostatistics and

Epidemiology, Graduate School of

Public Health, Seoul National University,

${ }^{\S}$ Korea University College of Health Sciences, Seoul,

${ }^{f}$ Dept of Nursing, Kwandong

University, Kangnung,

**Dept of Environmental, Civil

Engineering and Information System,

Seowon University,

\#\#Dept of Living Welfare, Seowon

University, Cheongju, and

"Dept of Social and Preventive

Medicine, Sungkyunkwan University School of Medicine, Suwon, South Korea.

CORRESPONDENCE

Y-C. Hong

Dept of Preventive Medicine, Seoul National University College of Medicine, 28 Yongon-Dong, Chongno-Gu, Seoul 110-799, South Korea

E-mail: ychong1@snu.ac.kr

Received:

Sept 292009

Accepted after revision:

Feb 182010

First published online:

March 292010

European Respiratory Journal

Print ISSN 0903-1936

Online ISSN 1399-3003 
city and a rural area, respectively. The elderly returned to their home environment when they were not at the day care facilities.

Repeated spirometric tests were performed up to eight times on different days for each subject. We also measured urinary concentrations of metabolites of the VOC and markers of oxidative stress on the same day as spirometric tests. However, three refused participation and four performed spirometric testing only once. Therefore, we included 154 subjects who had performed spirometric testing twice or more in the analysis.

At the first visit, information such as demographic factors, medical history, dietary habits, smoking and alcohol consumption was obtained by a questionnaire. The first voided urines were collected for biomarker measurements at each visit day.

Written informed consent was obtained from every subject, and the study was approved by the institutional review board of the Seoul National University College of Medicine, Seoul, South Korea.

\section{Spirometric measurements}

Spirometric testing was conducted four times in the warm season (June to September) and four times in the cold season (October to December) according to 2005 European Respiratory Society/American Thoracic Society recommendations. All tests were performed at the same hour in the morning by one trained technician, and a Microlab ${ }_{\mathbb{R}}$ (SensorMedics, Yorba Linda, CA, USA) spirometer was used.

The spirometric tests used in our analyses were forced vital capacity (FVC), forced expiratory volume in $1 \mathrm{~s}$ (FEV1), FEV1 as a percentage of FVC (FEV1/FVC), and forced expiratory flow at $25-75 \%$ of FVC (FEF25-75\%).

\section{Urinary levels of VOC metabolites}

On the same day of spirometric tests, tt-muconic acid, hippuric acid, mandelic acid and methylhippuric acid were measured as biomarkers for exposure to benzene, toluene, ethylbenzene and xylene, respectively. To determine the tt-muconic acid levels, $200 \mu \mathrm{L}$ of urine were mixed with $800 \mu \mathrm{L}$ of mobile phase ( $1 \%$ acetic acid and methanol, 10:1, v/v) and filtered through a $0.45-\mu \mathrm{m}$ membrane filter. Absorbance of the filtered solution was measured by HPLC-UV at $259 \mathrm{~nm}$ using a Shiseido MF C8 SG80 $5 \mu \mathrm{m}(4.6 \times 150 \mathrm{~mm})$ column (Tokyo, Japan). To determine the hippuric acid, mandelic acid and methylhippuric acid levels, $200 \mu \mathrm{L}$ of urine were mixed with $2 \mathrm{~mL}$ of distilled water and filtered through a $0.45-\mu \mathrm{m}$ membrane filter. Absorbance of the filtered solution was measured by HPLC-UV at $225 \mathrm{~nm}$ using Waters Atlantis C18 $5 \mu \mathrm{m}(4.6 \times 150 \mathrm{~mm})$ column (Milford, MA, USA). The mobile phase was $0.55 \%$ ntetrabutylammonium bromide $/ 0.15 \% \quad \mathrm{KH}_{2} \mathrm{PO}_{4}$ and methanol $(60: 40 v / v)$.

\section{Urinary levels of oxidative stress biomarkers}

For evaluation of oxidative stress, urinary malondialdehyde (MDA) was determined by the measurement of MDAthiobarbituric acid (TBA) adducts. Aliquots of the upper layer of the centrifuged urine samples were mixed with phosphoric acid and TBA reagent in an iced methanol glass tube, and were boiled for $60 \mathrm{~min}$. After the mixture was boiled, it was cooled in ice water for $5 \mathrm{~min}$ and centrifuged after adding methanol.
The absorbance was measured by an HPLC system equipped with a SP930D solvent delivery pump and UV730 absorbance detector (Youngjin company, Seoul, South Korea) on a NovaPak C18 column $(150 \times 3.9 \mathrm{~nm})$ (Waters) at $532 \mathrm{~nm}$ with a mobile phase composed of $50 \mathrm{nM} \mathrm{KH_{2 }} \mathrm{PO}_{4}(\mathrm{pH}$ 6.8) and methanol in a ratio of 58:42 v/v [14].

Urinary 8-oxo-2'-deoxyguanosine (8-OHdG) levels were quantitatively determined using a competitive in vitro ELISA (JICA, Fukuroi, Japan). $50 \mu \mathrm{L}$ of primary monoclonal antibody and $50 \mu \mathrm{L}$ of sample or standard were added to microtitre plates, which had been pre-coated with $8-\mathrm{OHdG}$. The plates were sealed tightly and incubated at $37^{\circ} \mathrm{C}$ for $1 \mathrm{~h}$, and then washed with $250 \mu \mathrm{L}$ PBS. $100 \mu \mathrm{L}$ of horseradish peroxidase-conjugated secondary antibody were then added to each well, incubated and washed. $100 \mu \mathrm{L}$ of enzyme substrate was then added to each well. Reactions were terminated by adding $100 \mu \mathrm{L} 1 \mathrm{~N}$ phosphoric acid. Absorbance readings were taken 3 min after termination using a spectrophotometer (ELX808; Bio-Tek, Winooski, VT, USA) at $450 \mathrm{~nm}$ [14].

\section{Ambient levels of VOC}

We measured indoor levels of benzene, toluene, ethylbenzene and xylene (once at two places) and outdoor levels (at one place) at the same time for each facility during the study period to compare ambient levels of the VOC with the levels of other reports. The sampling equipment used to collect the VOC was placed $1.5 \mathrm{~m}$ above floor level indoors and at ground level outdoors at the day care facilities. Air samples were collected by passing air through adsorbent tubes (Tenax, Eighty Four, PA, USA). The tubes were connected with air sampler (MP-100 H; Sibata Scientific Technology Ltd, Tokyo, Japan) for absorption of the VOC. The sampling rate was $100 \mathrm{~mL} \cdot \mathrm{min}^{-1}$ for $8 \mathrm{~h}(09.00 \mathrm{~h}$ to $17.00 \mathrm{~h})$ and the samples were analysed by GC/MSD (6890N, Agilent Technologies, Santa Clara, CA, USA) in a capillary column (60 m length, $0.25 \mathrm{~mm}$ inner diameter and $0.25 \mu \mathrm{m}$ film thickness) using Thermal Desorption System (Gerstel GmbH and Co. KG, Mülheim, Germany).

\section{Measurements of potential confounders}

Urinary cotinine concentrations

We measured urinary concentrations of cotinine because tobacco exposure was regarded as a confounder and cotinine is widely considered to be the best marker for monitoring tobacco exposure in either actively or passively exposed individuals. The urine samples were stored at $-20^{\circ} \mathrm{C}$ and analysed in batches. Urinary cotinine level was measured using a microplate enzyme immunoassay (UMAX, Dallas, TX, USA). The microplate was coated with an anti-cotinine antibody and cotinine concentration was determined by a competitive reaction with an enzyme-bound and non-enzyme-bound antigens. Reactions were detected at $450 \mathrm{~nm}$ using a VersaMax microplate reader (Molecular Devices, Sunnyvale, CA, USA).

\section{Personal exposure to PM2.5 and nitrogen dioxide}

We measured personal exposure to PM2.5 (particles with a 50\% cut-off aerodynamic diameter of $2.5 \mu \mathrm{m}$ ) in participating subjects. The particles were sampled around $24 \mathrm{~h}$ for each visit with a personal environmental monitor PM2.5 cyclon (SKC Inc., Washington, PA, USA) containing a pump (Mine Safety 
Appliances company, Pittsburgh, PA, USA). The air flow rate $\left(2 \mathrm{~L} \cdot \mathrm{min}^{-1}\right)$ of the pump was adjusted before each sampling. The PM2.5 cyclon was attached with a pin between the chest and head, and the pump was placed in a small bag, which was carried by the subjects during measurement. The PM2.5 cyclon and the pump were kept near the bedside during sleep. The $37 \mathrm{~mm}, 2.0 \mu \mathrm{m}$ Teflon filters (SKC, Eighty Four, PA, USA) were weighed before and after sampling using a microbalance and the concentration of ambient particulate matter was calculated considering air flow and measurement time.

Personal nitrogen dioxide $\left(\mathrm{NO}_{2}\right)$ exposures were also measured simultaneously with PM2.5. Polyester housed badge-type $\mathrm{NO}_{2}$ passive samplers (Envors Co., Daejon, South Korea) were used for the study. One of the investigators gave instructions on how to wear and care for the samplers. Unsealed passive sampling badges were attached with a pin between the chest and head, and were sealed the following day. Wearers were also instructed to keep the badges near the bedside when they slept. The collected samplers were sealed with a gas-tight polyester lid and kept in a zippered plastic bag before and after sampling until analysis. The ambient $\mathrm{NO}_{2}$ concentration was calculated by using a $\mathrm{NO}_{2}$ standard solution curve assuming a given sampling rate, i.e. $16.9 \mathrm{~mL} \cdot \mathrm{min}^{-1}$, which was supplied by the manufacturer.

\section{Temperature and humidity}

Data for outdoor temperature and relative humidity were obtained from the Korea Meteorological Administration, Seoul, South Korea.

\section{Statistical analysis}

We calculated the means and distribution percentiles for urinary concentrations of biomarkers. For concentrations below the limits of detection (LOD), a value equal to the LOD divided by two was used in the statistical analysis. We assessed associations between urinary metabolites of VOC, oxidative stress markers and pulmonary function parameters. Linear mixed-effect models were used to estimate the VOC exposure effects on the pulmonary function test (PFT) parameters or the oxidative stress markers, controlling for temperature, humidity, cotinine levels, dietary habits, alcohol consumption and individual characteristics. Personal exposures to PM2.5 and $\mathrm{NO}_{2}$ were also controlled in the statistical models. Because the distributions of biomarker concentrations were skewed, we used log-transformed data for these measurements in the linear mixed models. We treated age, sex, height, weight, date, temperature, relative humidity, cotinine levels, PM2.5 and $\mathrm{NO}_{2}$ exposure levels, dietary habits, alcohol consumption and biomarker concentrations as fixed effects. Each participant was treated as a random effect in the models. Because we had similar results regardless of adjustment for urine creatinine, we report the association results using non-adjusted ones for urinary levels of VOC metabolites and oxidative stress markers.

\section{RESULTS}

\section{Participant characteristics and biochemical analyses}

The participant mean age was $73.5 \mathrm{yrs}$ old and $63.0 \%$ were female. Of a possible total of eight visits, mean number of visits was 6.8 visits per person. The number of persons whose level of urinary cotinine was over $300 \mu \mathrm{g} \cdot \mathrm{L}^{-1}$ was $22(14.3 \%)$ (table 1$)$.
The indoor levels $(\mathrm{n}=12)$ of benzene, toluene, ethylbenzene and xylene were $5.96 \pm 4.08,11.18 \pm 10.49,4.55 \pm 1.78$ and $5.24 \pm 1.35 \mu \mathrm{g} \cdot \mathrm{m}^{-3}$, respectively. The outdoor levels $(\mathrm{n}=6)$ were $4.31 \pm 3.00,6.20 \pm 8.71,3.10 \pm 1.56$ and $2.84 \pm 1.49 \mu \mathrm{g} \cdot \mathrm{m}^{-3}$, respectively. The results of measurements for urinary markers of VOC metabolites, $\mathrm{NO}_{2}, \mathrm{PM} 2.5$, outdoor temperature and relative humidity are shown in table 2 . The percentage of measurements that were lower than limit of detection $(\%<\mathrm{LOD})$ are also shown. Among the measured biomarkers, the \% $<$ LOD of tt-muconic acid was $>50 \%$.

\section{Effects of VOC metabolites on lung function parameters}

The levels of hippuric acid were negatively associated with FEV1 and FEF25-75\%, and methylhippuric acid levels were negatively associated with FEV1 and FEV1/FVC (table 3). However, tt-muconic acid and mandelic acid were not significantly associated with either PFT parameters.

To estimate effects of VOC metabolites on lung function, we calculated the estimated values of lung function parameters assuming average values of age, sex, height, weight, temperature, humidity, cotinine level, $\mathrm{PM} 2.5$ and $\mathrm{NO}_{2}$ levels, dietary habits and alcohol consumption. As shown in figure 1, FEV1 is decreased by $21.5 \mathrm{~mL}(1.2 \%), \mathrm{FEV} 1 / \mathrm{FVC}$ by $0.5 \%(0.6 \%)$, FEF25-75\% by $74.6 \mathrm{~mL} \cdot \mathrm{s}^{-1}(3.6 \%)$ when the value of hippuric acid is at its 90 th percentile compared to its $10^{\text {th }}$ percentile. FEV1 decreases by $18.2 \mathrm{~mL}(1.0 \%), \mathrm{FEV} 1 / \mathrm{FVC}$ by $0.7 \%(0.7 \%)$, and FEF25-75\% by $40.5 \mathrm{~mL} \cdot \mathrm{s}^{-1}(1.9 \%)$ when methylhippuric acid is at its $90^{\text {th }}$ compared to its 10 th percentile. (fig. 1).

\section{Associations among VOC metabolites, oxidative stress and lung function}

Except for the tt-muconic acid, all of the other VOC metabolites were significantly associated with urinary MDA levels after adjustment for age, sex, height, weight, cotinine levels, PM2.5 and $\mathrm{NO}_{2}$ levels, dietary habits, alcohol consumption, temperature and humidity. All of the measured VOC metabolites were also significantly associated with urinary 8-OHdG (table 4).

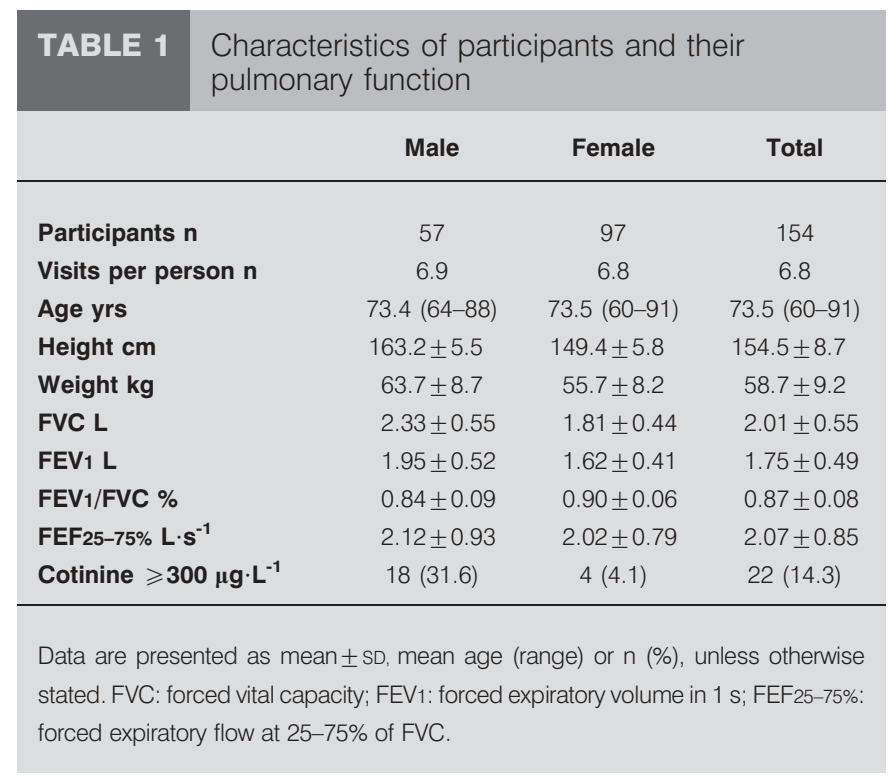


TABLE 2 Urinary concentrations and distributions of the volatile organic compound (VOC) metabolites, nitrogen dioxide, particles with a 50\% cut-off aerodynamic diameter of $2.5 \mu \mathrm{m}$ (PM2.5), outdoor temperature and relative humidity

\begin{tabular}{|c|c|c|c|c|c|c|c|c|c|}
\hline VOC metabolites & Measurements $\mathrm{n}$ & Mean \pm SD & LOD & $<$ LOD $\%$ & 10 pct & 25 pct & 50 pct & 75 pct & 95 pct \\
\hline Muconic acid $\mathrm{mg} \cdot \mathrm{L}^{-1}$ & 988 & $0.08 \pm 0.10$ & 0.014 & 79.96 & 0.05 & 0.05 & 0.05 & 0.05 & 0.27 \\
\hline Hippuric acid $\mathrm{mg} \cdot \mathrm{mL}^{-1}$ & 984 & $0.53 \pm 0.65$ & 0.020 & 0.30 & 0.06 & 0.14 & 0.32 & 0.65 & 1.84 \\
\hline Methylhippuric acid $\mathrm{mg} \cdot \mathrm{mL}^{-1}$ & 984 & $0.10 \pm 0.19$ & 0.004 & 25.81 & 0.00 & 0.00 & 0.02 & 0.08 & 0.47 \\
\hline $\mathrm{NO}_{2} \mathrm{ppb}$ & 1033 & $21.68 \pm 11.46$ & 0.30 & 0.0 & 8.71 & 12.36 & 19.70 & 28.80 & 43.30 \\
\hline $\mathrm{PM} 2.5 \mu \mathrm{g} \cdot \mathrm{m}^{-3}$ & 1021 & $33.38 \pm 22.45$ & & & 8.87 & 17.00 & 28.81 & 43.59 & 78.31 \\
\hline Outdoor relative humidity $\%$ & 1037 & $68.47 \pm 16.78$ & & & 42.3 & 58.3 & 72.6 & 80.9 & 90.8 \\
\hline
\end{tabular}

LOD: limit of detection; pct: percentile.

When we analysed the relationships between the oxidative stress biomarkers and lung function parameters, we found that urinary MDA levels were negatively associated with FEV1 and urinary 8-OHdG levels were negatively associated with FEV1/ FVC and FEF25-75\%. However, FVC was not associated with either the MDA or 8-OHdG levels. Figure 2 shows the possible pathway for the effects of toluene and xylene exposure on lung function through oxidative stress.

\section{Association between indoor levels of VOCs and their metabolites}

When we analysed the relationship between indoor VOC levels at the day care facilities and VOC metabolites in urine, we found that urinary hippuric acid and mandelic acid levels were significantly associated with indoor toluene levels (regression coefficient $\beta$ 0.009; $\mathrm{p}=0.003$ ) and ethylbenzene levels $(\beta$ 0.061; $\mathrm{p}<0.001)$ while there was no significant association of urinary tt-muconic acid $(\beta-0.001 ; \mathrm{p}=0.589)$ and methylhippuric acid ( $\beta$ 0.003; $p=0.683)$ with indoor levels of benzene and xylene, respectively, after regression analysis controlling for age, sex, height, weight, date, temperature, relative humidity, cotinine levels, $\mathrm{PM} 2.5$ and $\mathrm{NO}_{2}$ exposure levels, dietary habits and alcohol consumption.

\section{DISCUSSION}

Our study demonstrates that exposure to toluene and xylene may exert harmful effects on lung function and that oxidative stress could be involved in the pathogenesis in the elderly population. Most of the reported investigations into the health effects of air pollution have been targeted at outdoor air pollutants, such as ozone and particulate matter $[7,8,15,16]$. The importance of indoor air quality, however, is progressively increasing since people spend more time indoors than outdoors. Although VOCs also exist in the outdoor environment, the concentrations of VOCs are two- to five-fold higher indoors than outdoors, so they are generally regarded as "indoor" air pollutants [17, 18].

In this study, we found a significant association between urinary levels of hippuric acid and methylhippuric acid and decreased lung function. These are metabolites of toluene and xylene, respectively, which are widely used in our daily necessities, such as paints, varnishes, rubber and disinfectants. Although benzoic acid from dietary intake is known to be one of the main sources of urinary hippuric acid, significant association between ambient levels of toluene and urinary hippuric acid has been reported [19]. More than $90 \%$ of xylene is known to be metabolised to methylhippuric acid, so urinary levels of methylhippuric acid can be used as the total body burden of xylene [20].

TABLE 3 Estimated regression coefficients of urinary concentrations of the volatile organic compund (VOC) metabolites on lung function parameters

\begin{tabular}{|c|c|c|c|c|c|c|}
\hline \multirow[t]{2}{*}{ VOC metabolites } & \multicolumn{2}{|c|}{ FEV1 mL } & \multicolumn{2}{|c|}{ FEV $_{1} /$ FVC $_{0} \%$} & \multicolumn{2}{|c|}{$\mathrm{FEF} 25-75 \% \mathrm{~mL} \cdot \mathrm{s}^{-1}$} \\
\hline & Coefficient & $\mathrm{p}$-value & Coefficient & $\mathrm{p}$-value & Coefficient & $p$-value \\
\hline Hippuric acid $\mathrm{mg} \cdot \mathrm{mL}^{-1}$ & $-18.23 \pm 8.52$ & 0.033 & $-0.47 \pm 0.29$ & 0.107 & $-65.00 \pm 21.81$ & 0.003 \\
\hline Methylhippuric acid $\mathrm{mg} \cdot \mathrm{mL}^{-1}$ & $-65.70 \pm 30.41$ & 0.031 & $-2.44 \pm 1.00$ & 0.015 & $-145.85 \pm 76.46$ & 0.057 \\
\hline Mandelic acid $\mathrm{mg} \cdot \mathrm{mL}^{-1}$ & $-15.10 \pm 45.69$ & 0.741 & $-1.03 \pm 1.58$ & 0.514 & $-67.67 \pm 117.24$ & 0.564 \\
\hline
\end{tabular}

Data are presented as mean \pm SE, unless otherwise stated. FEV1: forced expiratory volume in 1 s; FVC: forced vital capacity; FEF25-75\%: forced expiratory flow at 25-75\% of FVC. p-values are from mixed regression analysis adjusted for visit number, age, sex, height, weight, outdoor temperature, outdoor relative humidity, diet, cotinine, alcohol consumption, levels of nitrogen dioxide and particles with a 50\% cut-off aerodynamic diameter of $2.5 \mu \mathrm{m}$. 

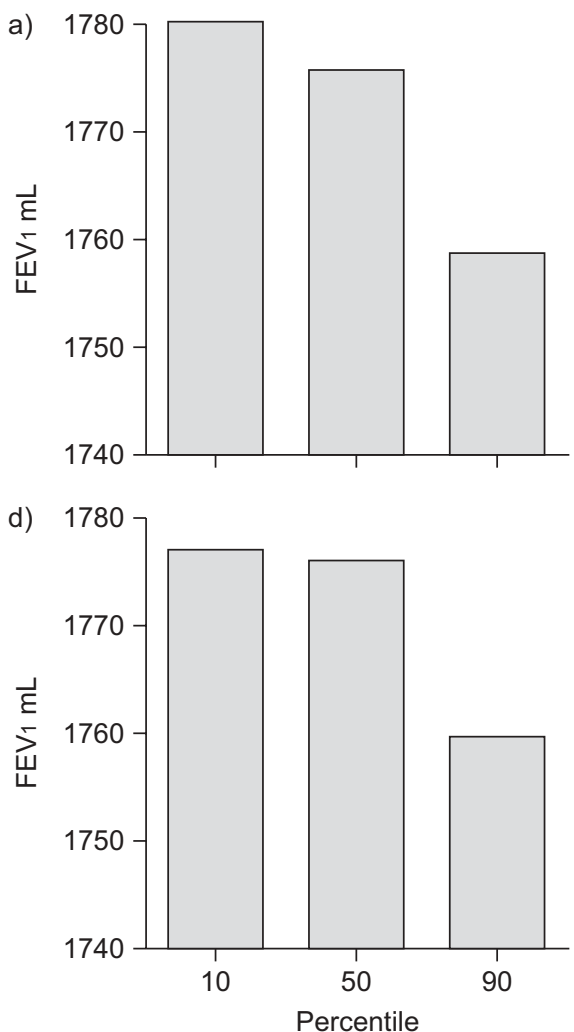
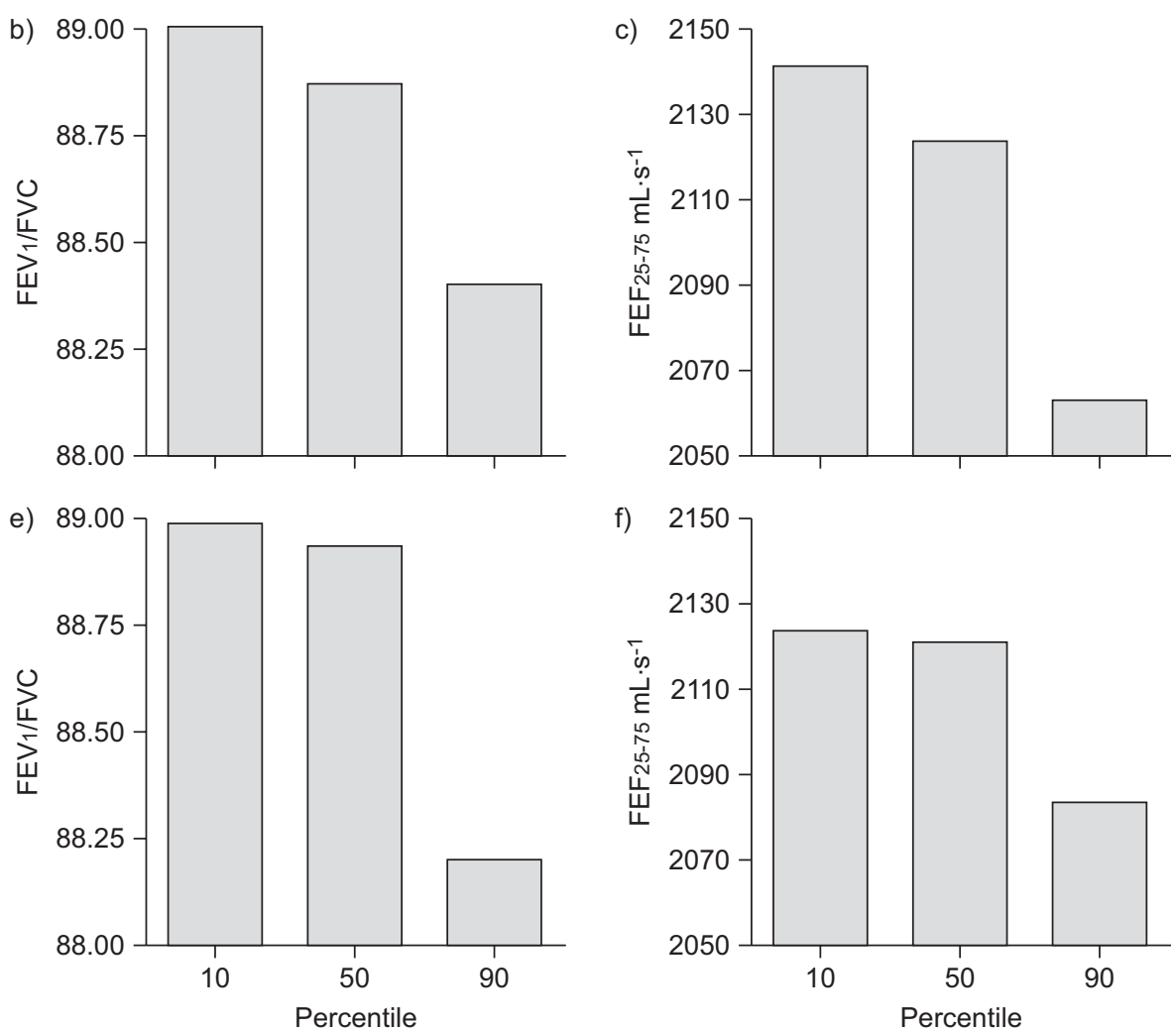

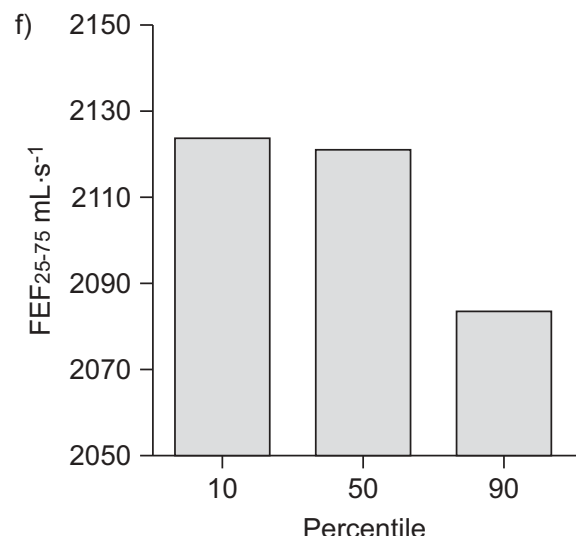

FIGURE 1. Estimated effects of urinary concentrations of a-c) hippuric acid and d-f) methylhippuric acid on lung function parameters. Each level of three pulmonary function parameters at the 10,50 and 90th percentile of urinary levels of hippuric acid and methylhippuric acid were estimated. Mean values of age, sex, height, weight, outdoor temperature, outdoor relative humidity, area, cotinine and alcohol consumption were used for estimation. FEV1: forced expiratory volume in $1 \mathrm{~S}$; FVC: forced vital capacity; FEF25-75\%: forced expiratory flow at $25-75 \%$ of FVC.

Muconic acid and mandelic acid, which were supposed to represent exposure to benzene and ethylbenzene, respectively, did not exhibit an association with lung function parameters. However, this could be due to the concentration under the

\begin{tabular}{|c|c|c|c|c|}
\hline \multirow[t]{2}{*}{ VOC metabolites } & \multicolumn{2}{|c|}{$\mathrm{MDA} \mu \mathrm{mol} \cdot \mathrm{L}^{-1}$} & \multicolumn{2}{|c|}{$8-\mathrm{OHdG} \mathrm{ng} \cdot \mathrm{mL}^{-1}$} \\
\hline & Coefficient & p-value & Coefficient & $p$-value \\
\hline Muconic acid $\mathrm{mg} \cdot \mathrm{L}^{-1}$ & $0.54 \pm 0.36$ & 0.132 & $8.23 \pm 2.51$ & 0.001 \\
\hline Hippuric acid $\mathrm{mg} \cdot \mathrm{mL}^{-1}$ & $0.51 \pm 0.06$ & $<0.001$ & $3.92 \pm 0.42$ & $<0.001$ \\
\hline $\begin{array}{l}\text { Methylhippuric acid } \\
\mathrm{mg} \cdot \mathrm{mL}^{-1}\end{array}$ & $0.84 \pm 0.21$ & $<0.001$ & $8.20 \pm 1.54$ & $<0.001$ \\
\hline $\begin{array}{l}\text { Mandelic acid } \\
\mathrm{mg} \cdot \mathrm{mL}^{-1}\end{array}$ & $2.20 \pm 0.32$ & $<0.001$ & $13.77 \pm 2.34$ & $<0.001$ \\
\hline
\end{tabular}

Data are presented as mean \pm SE, unless otherwise stated. MDA: malondialdehyde; 8-OHdG: 8-oxo-2'-deoxyguanosine. p-values are from mixed regression analysis adjusted for visit number, age, sex, height, weight, outdoor temperature, outdoor relative humidity, diet, cotinine, alcohol consumption, nitrogen dioxide and particles with a $50 \%$ cut-off aerodynamic diameter of $2.5 \mu \mathrm{m}$ levels. limit of detection $(80 \%$ and $42 \%)$ rather than a true nonassociation. As shown in professional painters or in sick building syndrome, exposure to an unusually high concentration of VOCs can result in respiratory effects [21,22]. Our panel consisted of elderly people without occupational exposure, so this study suggests that even ordinary exposure to VOCs can affect lung function, at least in the elderly population.

Of the measured parameters of lung function, FVC did not show any association with markers of VOC exposure in contrast to FEV1, FEV1/FVC, and FEF25-75\%. This suggests that the primary site of the pulmonary toxicity of VOCs might be the airways, especially the small airways rather than the pulmonary parenchyme.

The estimated changes of FEV1 were $21.5 \mathrm{~mL}$ for hippuric acid and $18.2 \mathrm{~mL}$ for methylhippuric acid, assuming the level of each metabolite changes to its 90th from the 10th percentile. Even though we assumed extreme circumstances, this is a relatively large change considering the annual loss of FEV1 in healthy nonsmokers in the general population is estimated to be $\sim 30 \mathrm{~mL} \cdot \mathrm{yr}^{-1}$ [23].

The proposed mechanism of VOC toxicity was oxidative stress [1-3], which is also thought to be the main mechanism of cigarette smoke-induced lung injury, acute exacerbation of chronic obstructive pulmonary disease, and many of the harmful effects of other air pollutants on respiratory epithelium [24-27]. The results of our study support the association 
VOC

VOC metabolites

Oxidative stress

biomarkers

Lung function

parameters

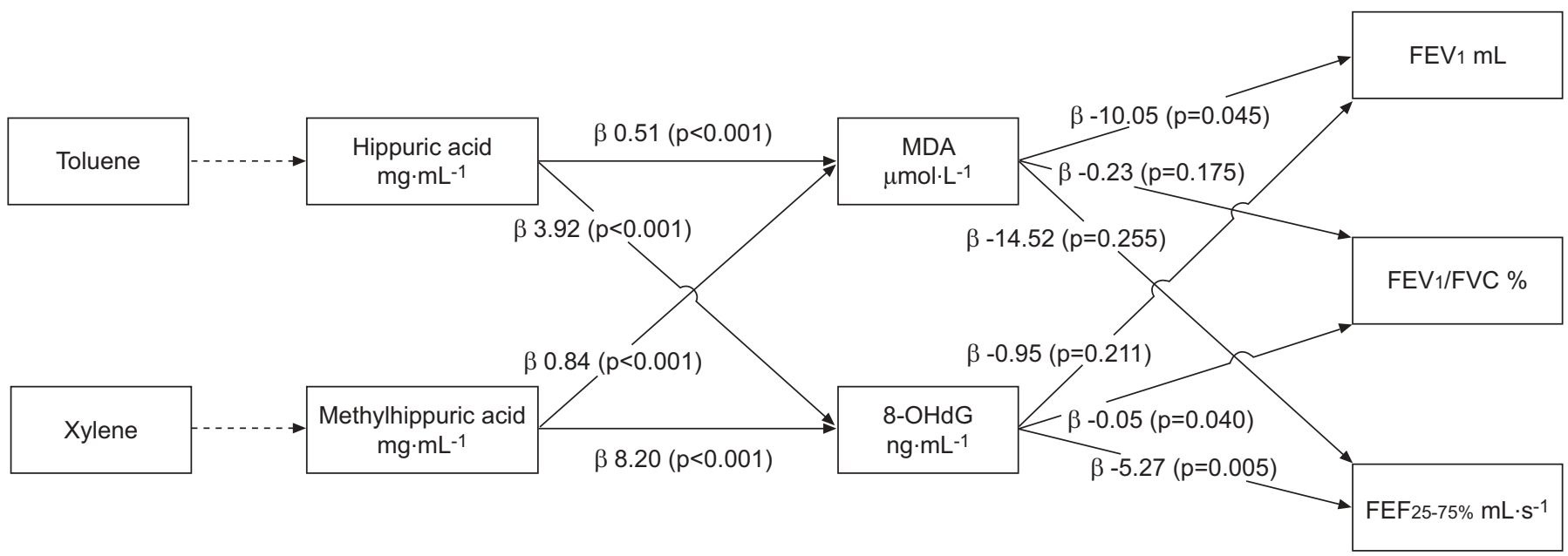

FIGURE 2. Possible pathway for the effects of toluene and xylene exposure on lung function through oxidative stress. VOC: volatile organic compound; MDA malondialdehyde; 8-OHdG: 8-oxo-2'-deoxyguanosine; FEV1: forced expiratory volume in 1 s; FVC forced vital capacity; FEF25-75\%: forced expiratory flow at 25-75\% of FVC.

between exposure to VOCs and increased systemic level of oxidative stress. Oxidative stress is also known to be influenced by many other factors, such as dietary intake, alcohol consumption and cigarette smoking, in addition to air pollution [19, 26-29]. In our data, however, significant associations between VOC metabolites and markers of oxidative stress were found even after adjustment for dietary intake, cotinine level, alcohol consumption, and PM2.5 and $\mathrm{NO}_{2}$ levels.

We also found a significant association between markers of oxidative stress and parameters of lung function. This is in line with the current understanding of the relationship of oxidative stress to a reduction in pulmonary function and increased cardiopulmonary mortality [20,30]. In addition, it supports the hypothesis that VOCs might influence lung function by exacerbating oxidative stress.

The strengths of the present study merit discussion. First, to the best of our knowledge, this is the first study to demonstrate the detrimental effects of toluene and xylene exposure on lung function in the course of ordinary environmental exposure. Secondly, the panel study design with repeated measurements of spirometric tests, urinary metabolites of VOCs and oxidative stress biomarkers up to eight times for each participant, provided a good opportunity to evaluate the short-term effects of changes in VOC exposure over time. Thirdly, by using biomarkers to evaluate personal exposures and outcome, this study demonstrated a possible pathway for the VOC effect, which suggests involvement of oxidative stress.

However, there are also some limitations to this study. We could not show associations between levels of indoor benzene and xylene and their metabolites. Because we only measured indoor levels of VOCs once at two spots in each day care facility, the data for the levels of indoor VOCs are thought to have failed to reflect personal variation of exposure and variation over time in contrast to those of the metabolites. The other reason for discrepancy between levels of indoor benzene and xylene and their metabolites could be that subjects were exposed to different levels in their home. Even though we adjusted for potential sources of oxidative stress in the statistical analyses, including personal exposure levels to PM2.5 and $\mathrm{NO}_{2}$, concomitant exposure to other pollutants, such as ozone and heavy metals, could have had some impact on lung function. Another concern relates to whether the VOC metabolites reflect true VOC exposure in the ordinary environmental circumstances. Although we controlled possible confounders, such as dietary habits, in the statistical model, we could not prove confidently that the VOC metabolites represent exposure to the chemicals in ambient air very well when the exposure levels are not high. Finally, we did not adjust levels of urinary biomarkers for levels of urine creatinine. All samples used in this analysis, however, were from the first morning urine in order to reduce the problem of dilution.

In conclusion, our findings suggest that exposure to toluene and xylene increase oxidative stress, which results in a deterioration of lung function in the elderly population.

\section{STATEMENT OF INTEREST}

None declared.

\section{ACKNOWLEDGEMENTS}

This study was supported by the Ministry of Environment, South Korea.

\section{REFERENCES}

1 Garcon G, Dagher Z, Zerimech F, et al. Dunkerque City air pollution particulate matter-induced cytotoxicity, oxidative stress and inflammation in human epithelial lung cells (L132) in culture. Toxicol In Vitro 2006; 20: 519-528.

2 Coleman CA, Hull BE, McDougal JN, et al. The effect of m-xylene on cytotoxicity and cellular antioxidant status in rat dermal equivalents. Toxicol Lett 2003; 142: 133-142.

3 Roder-Stolinski C, Fischader G, Oostingh GJ, et al. Styrene induces an inflammatory response in human lung epithelial cells via 
oxidative stress and NF-kappaB activation. Toxicol Appl Pharmacol 2008; 231: 241-247.

4 Hole DJ, Watt GC, Davey-Smith G, et al. Impaired lung function and mortality risk in men and women: findings from the Renfrew and Paisley prospective population study. BMJ 1996; 313: 711-715.

5 Mannino DM, Buist AS, Petty TL, et al. Lung function and mortality in the United States: data from the First National Health and Nutrition Examination Survey follow up study. Thorax 2003; 58: 388-393.

6 Sircar K, Hnizdo E, Petsonk E, et al. Decline in lung function and mortality: implications for medical monitoring. Occup Environ Med 2007; 64: 461-466.

7 Downs SH, Schindler C, Liu LJS, et al. Reduced exposure to PM10 and attenuated age-related decline in lung function. $N$ Engl J Med 2007; 357: 2338-2347.

8 Abbey DE, Burchette RJ, Knutsen SF, et al. Long-term particulate and other air pollutants and lung function in nonsmokers. Am J Respir Crit Care Med 1998; 158: 289-298.

9 Ackermann-Liebrich U, Leuenberger P, Schwartz J, et al. Lung function and long term exposure to air pollutants in Switzerland. Study on Air Pollution and Lung Diseases in Adults (SAPALDIA) Team. Am J Respir Crit Care Med 1997; 155: 122-129.

10 Elliott L, Longnecker MP, Kissling GE, et al. Volatile organic compounds and pulmonary function in the Third National Health and Nutrition Examination Survey, 1988-1994. Environ Health Perspec 2006; 114: 1210-1214.

11 Cakmak S, Dales RE, Vidal CB. Air pollution and mortality in Chile: susceptibility among the elderly. Environ Health Perspect 2007; 115: 524-527.

12 Fischer P, Hoek G, Brunekreef B, et al. Air pollution and mortality in The Netherlands: are the elderly more at risk? Eur Respir J Suppl 2003; 40: 34s-38s.

13 Medina-Ramon M, Schwartz J. Who is more vulnerable to die from ozone air pollution? Epidemiology 2008; 19: 672-679.

14 Kim YJ, Hong YC, Lee $\mathrm{KH}$, et al. Oxidative stress in pregnant women and birth weight reduction. Reprod Toxicol 2005; 19: 487-492.

15 Lippmann M. Health effects of airborne particulate matter. N Engl J Med 2007; 357: 2395-2397.

16 Oftedal B, Brunekreef B, Nystad W, et al. Residential outdoor air pollution and lung function in schoolchildren. Epidemiology 2008; 19: 129-137.

17 Sexton K, Adgate JL, Ramachandran G, et al. Comparison of personal, indoor, and outdoor exposures to hazardous air pollutants in three urban communities. Environ Sci Technol 2004; 38: 423-430.

18 Wallace LA. Personal exposure to 25 volatile organic compounds. EPA's 1987 team study in Los Angeles, California. Toxicol Ind Health 1991; 7: 203-208.

19 Zima T, Fialova L, Mestek O, et al. Oxidative stress, metabolism of ethanol and alcohol-related diseases. J Biomed Sci 2001; 8: 59-70.

20 Kelly FJ. Vitamins and respiratory disease: antioxidant micronutrients in pulmonary health and disease. Proc Nutr Soc 2005; 64: 510-526.

21 Wieslander G, Norback D, Edling C. Airway symptoms among house painters in relation to exposure to volatile organic compounds (VOCs)—a longitudinal study. Ann Occup Hyg 1997; 41: 155-166.

22 Talini D, Monteverdi A, Benvenuti A, et al. Asthma-like symptoms, atopy, and bronchial responsiveness in furniture workers. Occup Environ Med 1998; 55: 786-791.

23 Anthonisen NR, Connett JE, Kiley JP, et al. Effects of smoking intervention and the use of an inhaled anticholinergic bronchodilator on the rate of decline of FEV1. The Lung Health Study. JAMA 1994; 272: 1497-1505.

24 Drost EM, Skwarski KM, Sauleda J, et al. Oxidative stress and airway inflammation in severe exacerbations of COPD. Thorax 2005; 60: 293-300.

25 Sahin U, Unlu M, Ozguner F, et al. Lipid peroxidation and glutathione peroxidase activity in chronic obstructive pulmonary disease exacerbation: prognostic value of malondialdehyde. J Basic Clin Physiol Pharmacol 2001; 12: 59-68.

26 van der Toorn M, Smit-de Vries MP, et al. Cigarette smoke irreversibly modifies glutathione in airway epithelial cells. Am J Physiol Lung Cell Mol Physiol 2007; 293: L1156-L1162.

27 William A, Pryor KS. Oxidants in cigarette smoke radicals, hydrogen peroxide, eroxynitrate, and eroxynitritea. Ann $N$ Y Acad Sci 1993; 686: 12-27.

28 Rimm EB, Stampfer MJ, Ascherio A, et al. Vitamin E consumption and the risk of coronary heart disease in men. N Engl J Med 1993; 328: $1450-1456$.

29 Stampfer MJ, Hennekens $\mathrm{CH}$, Manson JE, et al. Vitamin E consumption and the risk of coronary disease in women. $N$ Engl J Med 1993; 328: 1444-1449.

30 Akbaraly TN, Favier A, Berr C. Total plasma carotenoids and mortality in the elderly: results of the Epidemiology of Vascular Ageing (EVA) study. Br J Nutr 2008; 29: 1-7. 